\title{
Explorer l'impact environnemental des transformations agraires en Asie du Sud-Est grâce à l'évaluation participative des services écosystémiques
}

\author{
Jean-Christophe Castella ${ }^{1, *}$ et Guillaume Lestrelin ${ }^{2,3}$ \\ 1 IRD, UMR GRED, UPVM, IRD, F-34000 Montpellier, France \\ 2 CIRAD, UMR TETIS, F-34398, Montpellier, France \\ 3 TETIS, Univ Montpellier, AgroParisTech, CIRAD, CNRS, INRAE, Montpellier, France
}

\begin{abstract}
Résumé - Différents mécanismes d'intervention sont proposés pour transformer les paysages agricoles de manière à ce qu'ils remplissent de multiples fonctions, compatibles avec les objectifs du développement durable. Dans le cas de l'atténuation et de l'adaptation au changement climatique, des politiques incitatives et des mécanismes de rémunération des acteurs locaux sont promues dans le cadre des initiatives REDD+. Sur le terrain, ces interventions visent à assurer simultanément la fourniture de services écosystémiques et le maintien, voire l'amélioration, des moyens d'existence locaux. Dans cet article, nous explorons le rôle que peut jouer l'évaluation participative dans la mise en œuvre de paiements pour services environnementaux au sein de communautés rurales d'Indonésie, du Laos, du Vietnam et de Chine. Engagées dans un processus d'intégration rapide à l'économie de marché, ces communautés ont transformé leurs systèmes d'abattisbrûlis traditionnels pour s'orienter vers différentes voies d'intensification agricole au cours des dernières années. L'évaluation positive ou négative de ces évolutions fait nécessairement l'objet de jugements de valeur dans les choix effectués entre différentes options possibles. La formulation de ces options en termes de services écosystémiques rendus permet de comparer les trajectoires paysagères et leur impact sur les conditions de vie locales. Sur ces bases, les communautés peuvent explorer des scénarios de transformation de leurs pratiques agricoles, négocier des compromis entre services écosystémiques et identifier les « gagnants » et les «perdants » potentiels. Ces simulations ont montré l'importance du calendrier de mise en place des programmes REDD + par rapport aux transformations agraires en cours. Elles pointent le risque de s'éloigner des ambitions initiales d'un impact sur les émissions de carbone pour devenir un instrument supplémentaire de développement durable.
\end{abstract}

Mots clés : services écosystémiques / simulation participative / changement agraire / REDD+ / Asie du Sud-Est

\begin{abstract}
Exploring the environmental impact of agrarian changes in Southeast Asia through participatory evaluation of ecosystem services. Different intervention mechanisms are proposed to transform agricultural landscapes so that they fulfill multiple functions, compatible with sustainable development goals. In the case of climate change mitigation and adaptation, support policies and direct payments to affected populations are promoted as part of REDD + initiatives. These interventions attempt to balance the provision of multiple ecosystem services with the maintenance or improvement of local livelihoods. In this paper, we explore the role that participatory evaluation can play in the implementation of payments for ecosystem services in rural communities in Indonesia, Laos, Vietnam and China. Engaged in a process of rapid integration into the market economy, these communities have been transforming their traditional Slash-and-burn systems in recent years and followed different paths of agricultural intensification. The positive or negative assessment of these developments is necessarily subject to value judgements in the choices made between different options. Ecosystem services allow for the comparison of landscape trajectories. Village communities explored scenarios for the evolution of their local agriculture, negotiated trade-offs between ecosystem services and identified potential "winners" and "losers". These simulations showed the importance of the timing of REDD+ programs in relation to
\end{abstract}

\footnotetext{
*Auteur de correspondance : j.castella@ird.fr
} 
ongoing agrarian transformations. They point to the risk of moving away from the initial ambitions related to the carbon ecosystem service to become an additional instrument for sustainable development.

Keywords: ecosystem services / participatory simulation / agrarian change / REDD+ / Southeast Asia

\section{Introduction}

Depuis plus de dix ans de mise en œuvre des mécanismes de réduction des émissions de gaz à effet de serre issus de la déforestation et de la dégradation forestière (REDD+; Cerbu et al., 2009; Barbier et Tesfaw, 2012; Sills et al., 2014), il existe peu de preuves empiriques sur la façon dont ces paiements pour services environnementaux (PSE) REDD + ont amélioré les conditions de vie et la sécurité alimentaire des acteurs locaux, notamment des populations les plus vulnérables (Jindal et al., 2008; Angelsen et al., 2019). Au-delà du bénéfice attendu sur la séquestration du carbone, il est essentiel d'explorer les impacts de la REDD+ sur les moyens d'existence et les autres co-bénéfices attendus, tels que la protection de la biodiversité (Grainger et al., 2009; Wunder et Wertz-Kanounnikoff, 2009). La perspective d'un paiement a poussé à formaliser une répartition des services écosystémiques en relation avec les attentes de différents groupes d'acteurs: carbone et biodiversité à l'échelle mondiale, régulation des flux d'eau et fertilité des sols pour les acteurs nationaux, sécurité alimentaire et augmentation des revenus agricoles pour les populations locales. La participation des différents acteurs à la conception de mécanismes d'intervention adaptés à leur situation est censée garantir que les intérêts des uns et des autres sont préservés (Leggett et Lovell, 2012). Ces intérêts ont rapidement dépassé la sphère forestière pour toucher plus largement la gestion des terres, y compris agricoles (Matthews et Van Noordwijk, 2014).

Dans le cas de la REDD+, la séquestration du carbone est vue comme un service écosystémique qui doit être rémunéré pour ceux qui le fournissent: agriculteurs, forestiers, etc. En principe, les usagers des terres sont indemnisés pour la conservation des forêts et l'amélioration des stocks de carbone, au-delà de leurs coûts d'opportunité. Le calcul des coûts d'opportunité permet d'évaluer le niveau de compensation que les mécanismes REDD + devraient fournir aux bénéficiaires. Cette première étape de l'évaluation de faisabilité de la REDD+ (Lestrelin et al., 2019) montre qu'il y a peu de chance que les crédits carbone à leur valeur internationale compensent le coût d'opportunité de la conversion de terres exploitées en abattis-brûlis (principalement des jeunes jachères et forêts secondaires) en grandes cultures commerciales comme l'hévéa (Bruun et al., 2018), le palmier à huile ou le maïs, principaux moteurs économiques de déforestation en Asie du Sud-Est (Mertz et al., 2009; Schmidt-Vogt et al., 2009; Cramb et al., 2009). De plus, des coûts de transaction se rajoutent aux coûts d'opportunité (Sills et al., 2014), et les questions de gouvernance constituent des obstacles majeurs à la mise en œuvre de la REDD+ sur le terrain (Wunder et al., 2008; Minang et al., 2014; Angelsen et al., 2019).

Une étude portant sur 80 communautés de dix pays d'Asie, d'Afrique et d'Amérique latine a montré que la participation de la population rurale dans les dispositifs de paiement pour service environnemental (PSE) a un impact positif sur le stockage du carbone (Chhatre et Agrawal, 2009). De même, certains chercheurs soutiennent que les populations locales sont, dans certains cas, des gestionnaires efficaces des mosaïques paysagères complexes qui intègrent l'essentiel des biomasses forestières (Danielsen et al., 2013; Brofeldt et al., 2014). En sus de la compensation financière, un des critères de succès de la REDD+ pourrait donc être sa capacité à intégrer les points de vue et les intérêts des populations locales dans la conception de mécanismes d'intervention qui ont du sens pour elles. Pour impliquer les futurs bénéficiaires dans des mécanismes de PSE, il est donc essentiel de rendre visible les avantages, les contraintes et les impacts potentiels, notamment dans le cadre d'un processus de consentement préalable libre et éclairé (Pham et al., 2015).

Dans cet article, nous présentons une étude comparative menée sur quatre terrains qui étaient encore caractérisés au début des années 2000 par des mosaïques paysagères complexes typiques des cultures sur abattis-brûlis (Lestrelin et al., 2019; Müller et al., 2014). Les sites d'étude (Fig. 1) sont situés dans la préfecture de Xishuangbanna de la province du Yunnan, en Chine (1); dans le district de Hiem, province de Huaphan, au Laos (2); dans le district de Con Cuong, province de Nghe An, au Vietnam (3); et dans le district de Kutai Barat, Kalimantan Est, Indonésie (4). Ils couvrent une grande diversité de milieux agroécologiques, de situations socioéconomiques et de modes de gouvernance forestière. L'histoire agraire des différents sites est présentée dans Müller et al. (2014) et Lestrelin et al. (2019). Sur la base de cette même étude de terrain, de précédents articles ont abordé la question des scénarios de référence et l'utilisation de contrefactuels (Müller et al., 2014), ainsi que le calcul des coûts d'opportunité et de leur rôle dans la définition des mécanismes de PSE (Lestrelin et al., 2019). Ici, nous nous concentrons sur la perception par les populations locales des services écosystémiques reçus ou attendus de différents types d'usage des terres agricoles et forestières. Après une présentation de l'approche utilisée pour caractériser les dynamiques d'usage des terres entre 2000 et 2012 dans les quatre sites d'étude, nous explorons avec les communautés locales les impacts perçus d'un éventuel mécanisme de PSE sur les paysages et les moyens d'existence.

\section{Méthodes}

\section{1 Évaluation participative des services écosystémiques associés aux systèmes de production et aux paysages agricoles}

Dans chacun des huit villages d'étude (deux villages par site), deux semaines ont été consacrées à la collecte d'un large éventail de données qualitatives et quantitatives par le biais d'une combinaison: (i) d'entretiens individuels utilisant des questionnaires semi-structurés sur 50 ménages sélectionnés de manière aléatoire dans chaque communauté villageoise ; (ii) de 


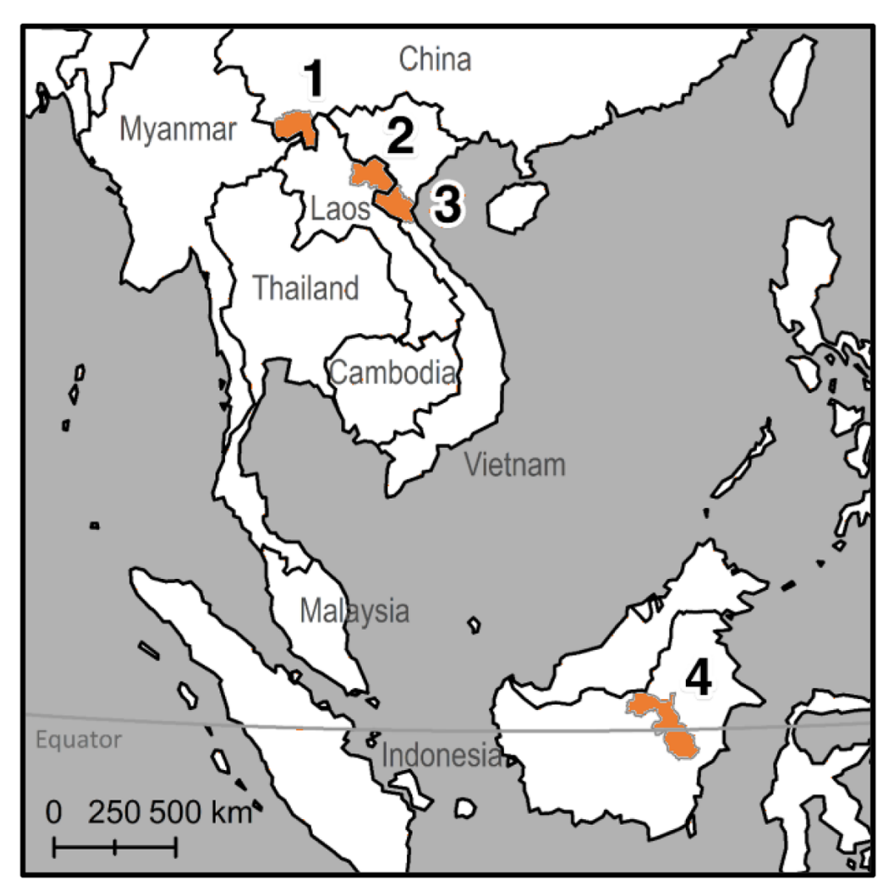

Fig. 1. Zones d'étude en Asie du Sud-Est.

Fig. 1. Study areas in Southeast Asia.

discussions de groupe impliquant plusieurs catégories d'acteurs locaux (agriculteurs, commerçants, chef de village, etc.), et (iii) de simulations participatives des dynamiques d'usage des terres et de leurs impacts sur les moyens d'existence. Sur la base des données recueillies, des typologies de ménages ont été produites à l'aide d'une méthode statistique combinant une analyse en composantes principales et une classification ascendante hiérarchique. Quatre systèmes de production différents ont été identifiés dans chaque village, puis validés avec les acteurs locaux. De façon générale, ces systèmes de production reflètent les situations suivantes :

- (A): essarteurs dans une logique de subsistance, qui pratiquent essentiellement des cultures sur abattis-brûlis ;

- (B) : riziculteurs qui atteignent l'autosuffisance en riz grâce à la combinaison de cultures extensives sur pentes et de rizières de bas-fonds ;

- (C) : agriculteurs qui accumulent du capital par le bétail ou les cultures pérennes, une fois la sécurité alimentaire atteinte sur la base des cultures de bas-fond et de pentes;

- (D) : agriculteurs mettant en œuvre des systèmes diversifiés combinant activités agricoles et non agricoles, grâce à l'investissement des revenus agricoles dans des activités non agricoles (Castella et al., 2013).

Pour chaque village, nous avons calculé le pourcentage de ménages correspondant à chacun des quatre types ci-dessus.

Les différents usages des terres ont ensuite été caractérisés de façon à prendre en compte les calendriers culturaux, les besoins en main-d'œuvre, la quantité d'intrants (semences, pesticides, etc.) et les rendements. Trois activités principales ont été prises en compte : les cultures, l'élevage et la collecte de produits forestiers non ligneux. De là, la productivité du travail et la productivité de la terre ont été calculées pour chaque usage des terres, et la valeur actuelle nette a été évaluée sur une période de 30 ans. Une méthode de vote distributif (Sheil et Liswanti, 2006) a ensuite été utilisée pour évaluer de manière participative la valeur perçue de chaque type d'usage des terres en termes de contribution aux moyens d'existence et aux services écosystémiques. Deux groupes (hommes et femmes) de dix personnes représentatives des différents types de systèmes de production identifiés ont été invités à évaluer la valeur perçue de chaque type d'utilisation des terres en distribuant dix grains de maïs chacun sur la liste de services suivante: sécurité alimentaire, revenus monétaires, santé humaine, culture et traditions, aménités esthétiques, régulation des eaux, conservation des sols, préservation de la biodiversité, qualité de l'air et du climat (Fig. 2). Cette liste de services écosystémiques utilisée par les chercheurs a été systématiquement adaptée et traduite selon les contextes locaux des quatre sites d'étude, afin qu'elle ait du sens et que les acteurs locaux qui ont participé aux évaluations se l'approprient pleinement. Les perceptions des hommes et des femmes ont ensuite été agrégées et pondérées par l'importance relative accordée par les villageois aux différents services écosystémiques.

\section{2 Évaluation de l'impact des dynamiques paysagères sur les services écosystémiques}

Des ateliers participatifs ont ensuite été organisés dans chacun des villages pour évaluer l'impact des changements d'usage des terres sur les services écosystémiques. Un groupe représentatif de la communauté villageoise (c'est-à-dire 12 à 15 personnes sélectionnées de manière à ce que le genre, les catégories d'âge, les quatre types de systèmes de production et l'appartenance ethnique soient représentés) a été constitué dans chaque village et engagé à cartographier l'usage des terres en 2000 et 2012 (année de réalisation des ateliers participatifs), en utilisant une maquette 3D du territoire villageois (Fig. 3). Les surfaces correspondant à différents usages des terres ont été estimées sur la base des cartes participatives, et des matrices de transition ont été calculées entre les deux dates (Fig. 3). En combinant l'évaluation participative des services écosystémiques décrite ci-dessus et les répartitions de l'usage des terres de 2000 et 2012, des «scores paysagers» ont été calculés pour 2000 et 2012 dans chaque village d'étude. Les différences entre les structures paysagères de 2000 et de 2012 pour différentes fonctions ou services ont ainsi servi d'indicateur d'impact du changement d'usage des terres sur les moyens d'existence et l'environnement. Il est en effet attendu d'un projet de PSE qu'il maintienne, voire accroisse, à la fois les moyens d'existence et les valeurs environnementales des paysages pour atteindre ses objectifs vis-à-vis des populations locales.

\subsection{Simulations participatives de mécanismes de paiement pour service environnemental}

Dans le contexte de nos terrains d'étude, les projets de PSE REDD + s'appuient sur des plans d'aménagement et de développement territorial intégrant: (i) la démarcation et la protection des forêts de conservation; (ii) l'intensification agroécologique pour accroître la productivité agricole tout en évitant les impacts négatifs d'un accès restreint aux ressources 

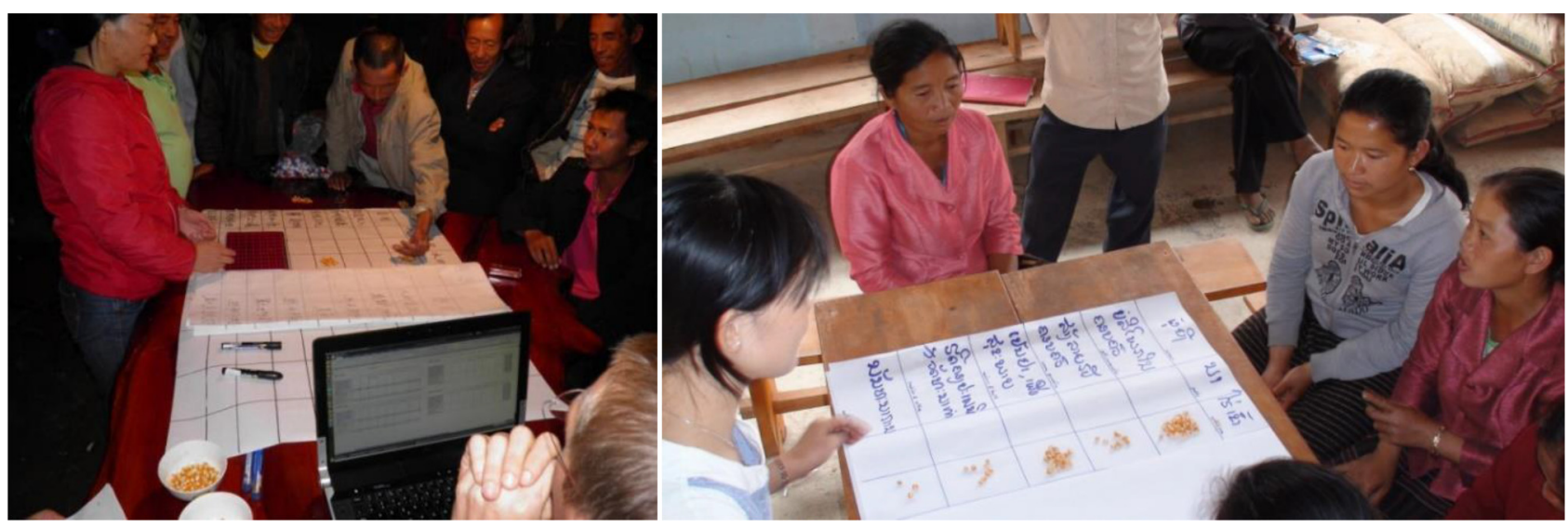

Fig. 2. Ateliers d'évaluation participative des services écosystémiques associés à différents types d'usage des terres.

Fig. 2. Participatory workshops on evaluation of ecosystem services associated with different land use types.

forestières sur les moyens d'existence locaux; et (iii) la plantation d'arbres pour augmenter la biomasse totale du paysage au-delà de la régénération naturelle des aires protégées (Lestrelin et al., 2013; Pham et al., 2019). Le plan d'aménagement du territoire est la pierre angulaire de la mise en œuvre de la REDD + car il détermine la répartition spatiale des différents types d'usages des terres, et donc les potentialités du territoire villageois en termes de génération de revenus et de fourniture de services écosystémiques tels que la séquestration du carbone et la préservation de la biodiversité.

Tout d'abord, les membres du groupe sont formés à négocier des plans d'utilisation des terres à partir d'un jeu de rôle réalisé sur un plateau de jeu de 100 cellules constituant un village virtuel, chaque cellule représentant 1 hectare de terre (Fig. 4). A travers la session de jeu, les participants apprennent comment la composition du paysage (pourcentage de cellules associées à chaque type d'utilisation du sol) détermine le potentiel économique du village ainsi que ses valeurs en carbone et en biodiversité. Ils négocient des compromis entre les différentes compositions du paysage, afin que les besoins et les exigences de différents intervenants (c'est-à-dire organismes de développement, organismes de protection des forêts et différents types de producteurs) puissent être satisfaits (Bourgoin et al., 2012, 2013 ; Castella et al., 2014).

Les participants appliquent ensuite la même méthode sur la maquette 3D de leur territoire villageois. À la fin de chaque cycle de négociation, les résultats en termes de distribution spatiale de l'usage des terres sont géo-référencés et numérisés dans un Système d'information géographique (SIG). Les impacts sur le revenu des différentes catégories de producteurs, les besoins en main-d'œuvre, la séquestration du carbone et la préservation de la biodiversité sont calculés sur la base du zonage (Fig. 4). Après plusieurs cycles de négociation et de discussion des résultats, les participants obtiennent un plan d'usage des terres consensuel. Des «crédits carbone» sont calculés en fonction de la différence entre la biomasse aérienne obtenue dans le cadre d'un scénario REDD+, et celle d'un scénario statu quo correspondant à une évolution naturelle de la biomasse sur une période d'une dizaine d'années.

\section{Résultats}

\subsection{Une décennie de transformations radicales des paysages agraires}

Au début des années 2000, le riz pluvial était la base de l'activité agricole et de l'alimentation dans tous les villages étudiés. Associé à du manioc, du taro, du maïs, des cucurbitacées ou des légumineuses, il occupait une place centrale dans des parcelles de culture en rotation longue avec des jachères de 12 à 15 ans. Dans la région chinoise du Xishuangbanna, comme au Laos et au Vietnam, les villageois pratiquaient également l'élevage de bovins et bubalins, caprins, porcins et volailles, pour leur propre consommation. Partout où les terres alluviales pouvaient être terrassées, les agriculteurs produisaient du riz de bas-fonds. La riziculture et l'élevage étaient moins développés dans l'est de Kalimantan; les villageois étaient engagés dans des systèmes agroforestiers à base d'hévéa et de rotin en combinaison avec des jardins fruitiers mixtes. L'agriculture était pratiquée dans des mosaïques de paysages complexes caractérisées par une intensité d'utilisation des terres décroissante en fonction de la distance aux zones habitées. Les villages étaient intégrés dans une matrice forestière et leurs limites n'étaient pas clairement définies. Les villageois dépendaient dans une large mesure des produits forestiers non ligneux pour leur subsistance et leur sécurité alimentaire en cas de mauvaises récoltes.

Dans tous les paysages étudiés, un processus généralisé d'intensification de l'usage des terres, c'est-à-dire un raccourcissement des périodes de jachère et/ou un allongement des périodes de culture, jusqu'à la culture permanente, a été observé durant la dernière décennie. Ce processus, impulsé (i) par les politiques foncières et les réformes foncières dans les années 1990, puis (ii) par l'intégration à l'économie de marché dans les années 2000, a été associé à une expansion rapide de l'agriculture commerciale et à une réduction progressive, voire à l'abandon, des pratiques traditionnelles d'abattis-brûlis. Ce virage de grande ampleur vers des cultures de vente (Lestrelin et al., 2019) a été facilité par le développement rapide des infrastructures de transport et de communication (Müller et al., 2014). La trajectoire d'intensification agricole décrite sur nos quatre terrains 

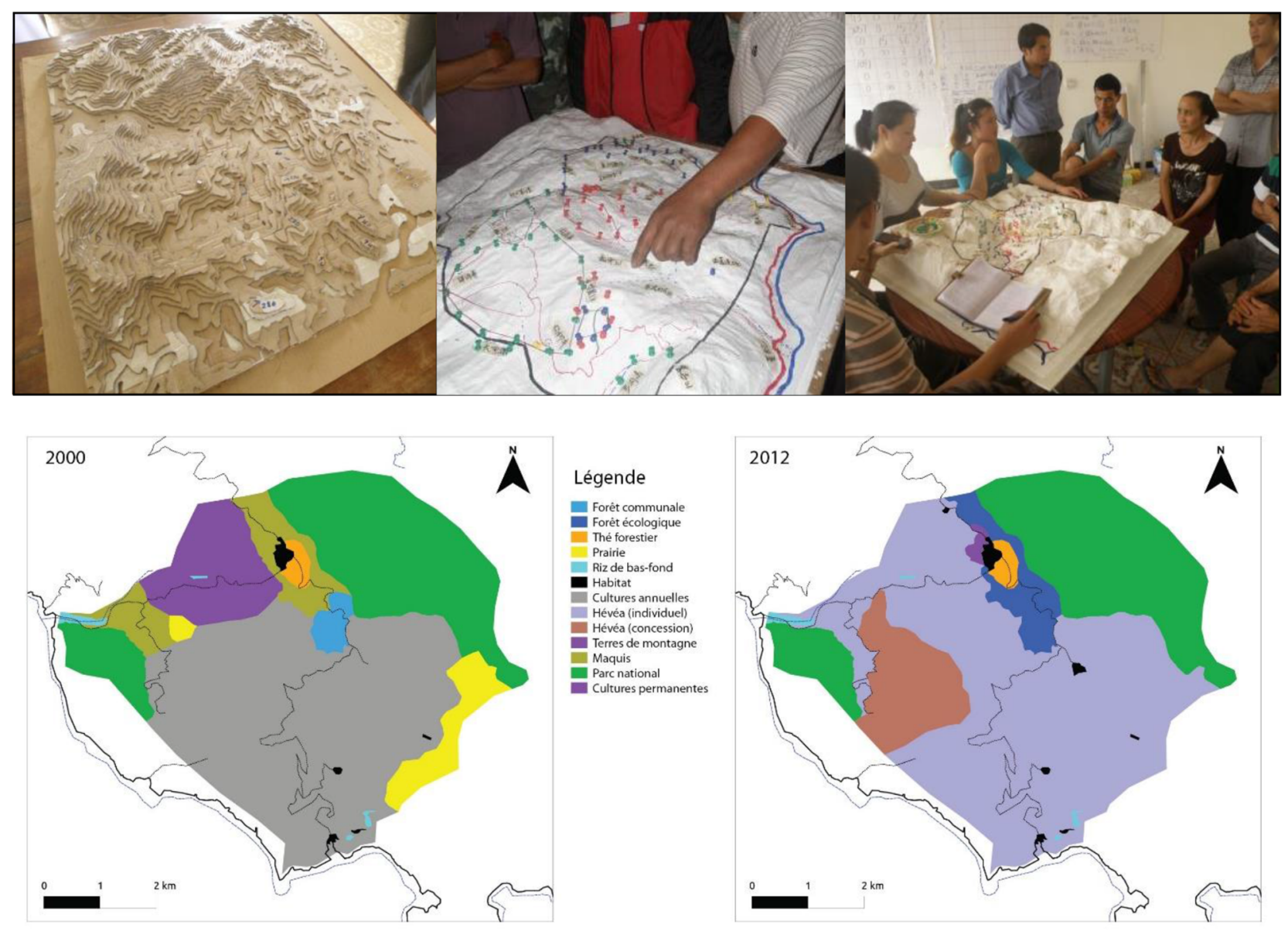

\begin{tabular}{|c|c|c|c|c|c|c|c|c|c|c|c|c|c|c|}
\hline $2000-2012$ & $\begin{array}{c}\text { Forêt } \\
\text { communale }\end{array}$ & $\begin{array}{c}\text { Forêt } \\
\text { protégée }\end{array}$ & Thé forestier & Prairie & $\begin{array}{l}\text { Riz de bas- } \\
\text { fond }\end{array}$ & Habitat & $\begin{array}{c}\text { Cultures } \\
\text { annuelles }\end{array}$ & $\begin{array}{c}\text { Hévéa } \\
\text { (individuel) }\end{array}$ & \begin{tabular}{|c|} 
Hévéa \\
(concession)
\end{tabular} & $\begin{array}{l}\text { Terres de } \\
\text { montagne }\end{array}$ & Maquis & $\begin{array}{c}\text { Parc } \\
\text { national }\end{array}$ & \begin{tabular}{|c|} 
Cultures \\
permanentes
\end{tabular} & Total \\
\hline Forêt communale & 0 & 57 & & & & & & & & & & & & 57 \\
\hline Forêt protégée & & 0 & & & & & & & & & & & & 0 \\
\hline Thé forestier & & & 29 & & & & & & & & & & & 29 \\
\hline Prairie & & & & 0 & & & & 155 & 13 & & & 11 & & 180 \\
\hline Riz de bas-fond & & & & & 17 & & & & & & & & & 17 \\
\hline Habitat & & & & & & 19 & & & & & & & & 19 \\
\hline Cultures annuelles & & & & & & 4 & 0 & 1423 & 286 & & & & & 1714 \\
\hline Hévéa (individuel) & & & & & & & & 0 & & & & & & 0 \\
\hline Hévéa (concession) & & & & & & & & & 0 & & & & & 0 \\
\hline Terres de montagne & & & & & & & & & & 0 & & & & 0 \\
\hline Maquis & & 106 & & & & 0,1 & & 96 & 20 & 17 & 0 & & & 239 \\
\hline Parc national & & & & & & & & & & & & 914 & & 914 \\
\hline Cultures permanentes & & & & & & 1 & & 331 & 11 & & & & 0 & 343 \\
\hline Total & 0 & 163 & 29 & 0 & 17 & 24 & 0 & 2005 & 330 & 17 & 0 & 925 & 0 & 3511 \\
\hline
\end{tabular}

Fig. 3. Cartographie participative de l'usage des terres dans le village de Manlin, Chine. En haut : sur un modèle 3D du village ; au centre : cartes d'usage des terres; en bas: matrice de changement d'usage des terres.

Fig. 3. Participatory land use mapping in Manlin Village, China. Top: on a 3D model of the village landscape; center: land use maps; bottom: land use change matrix.

d'étude reflète une dynamique de transformation agricole débutée dans les années 1980 en Thaillande avant sa diffusion régionale ultérieure, avec adaptations locales, au gré des réformes entreprises dans les pays voisins.

\subsection{Impacts des dynamiques d'usage des terres sur les services écosystémiques}

Les simulations participatives du changement d'usage des terres ont permis d'évaluer:
- les impacts de la transition d'une agriculture de subsistance à une agriculture commerciale sur les moyens d'existence et les services écosystémiques entre 2000 et 2012 ;

- les impacts potentiels qu'auraient eu des projets REDD+ mis en place entre 2000 à 2012 ;

- les impacts attendus d'un éventuel projet REDD+ qui aurait été mis en place entre 2012 et 2024. Le tableau 1 montre les résultats de la comparaison des cartes d'usage des terres pour chacun de ces trois scénarios en termes (i) de pourcentage de la superficie totale des terres dont l'utilisation a changé, et (ii) de changement de revenu total du village. 

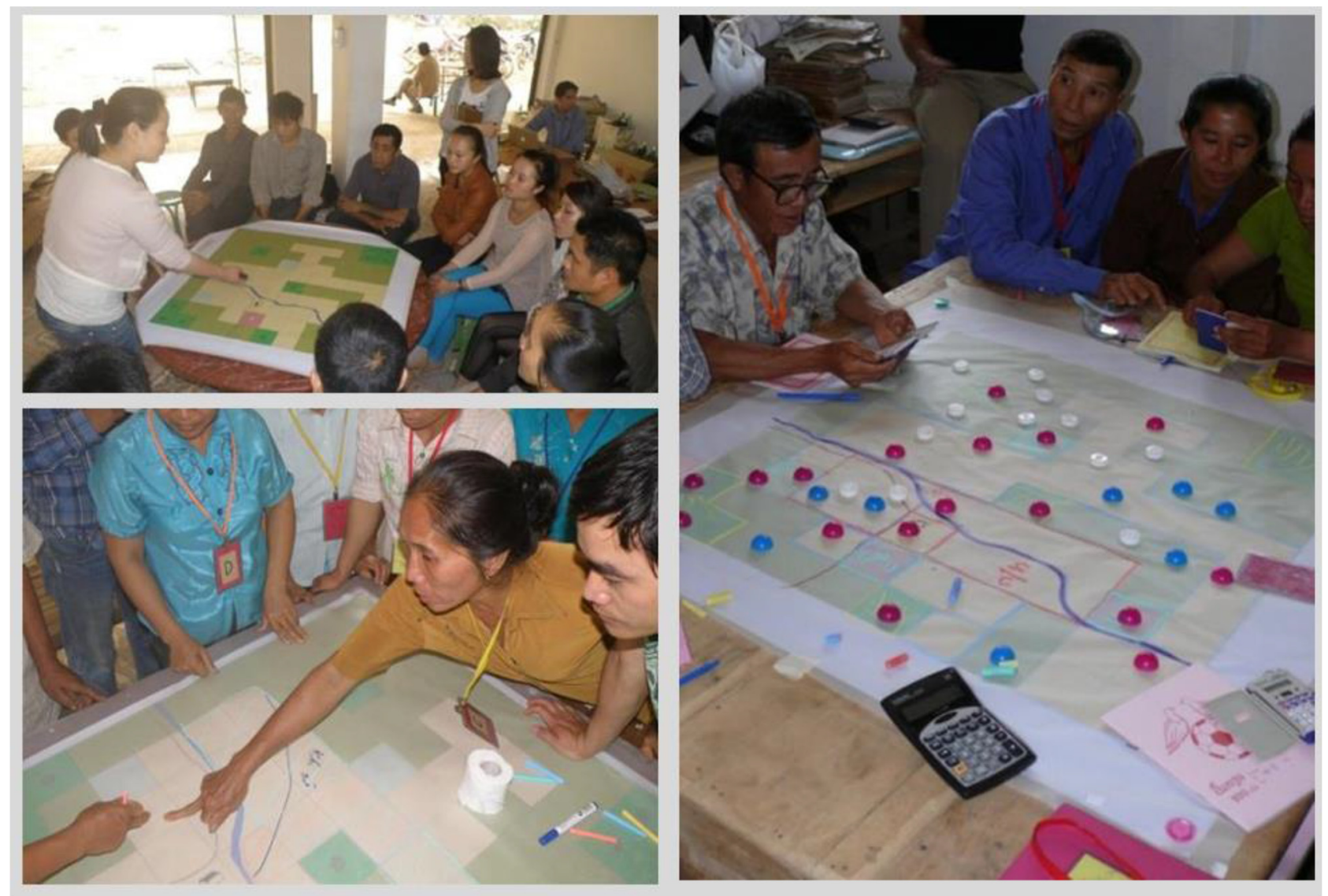

Fig. 4. Jeu de rôle utilisé pour la planification et simulation participative de l'usage des terres.

Fig. 4. Role-playing game used for participatory planning and simulation of land use.

Les grands changements d'affectation des terres observés dans les villages chinois $(75 \%)$, laotien $(81 \%)$ ou dans les deux villages du Vietnam $(47 \%$ et $56 \%)$ sont liés à une expansion rapide des cultures de rente, combinée à des restrictions réglementaires de l'accès des populations locales aux terres forestières. L'accès limité aux ressources forestières a eu un impact clairement négatif sur les modes de vie, tandis que les modèles de développement tirés par les opportunités du marché ont eu un impact positif sur les revenus des villageois, notamment dans les sites d'étude chinois et indonésiens (Tab. 1).

Les impacts perçus des changements d'usage des terres entre 2000 et 2012 ont généralement été négatifs en termes de sécurité alimentaire (Tab. 2). Dans le cas du Laos et du Vietnam, la perte d'accès aux ressources forestières n'a été que partiellement compensée par une augmentation des revenus issus de produits commercialisés. Les habitants des villages laotiens ont toutefois perçu une amélioration générale des services écosystémiques (biodiversité, qualité de l'air en relation avec les restrictions imposées sur les brûlis, etc.) liée à la mise en défens des zones forestières. La culture commerciale d'hévéa a augmenté considérablement la richesse des villages chinois, alors que les populations locales ont peu bénéficié de l'expansion du palmier à huile dans les villages indonésiens du fait d'un mode de production en concessions agro-industrielles. En Chine, l'impact environnemental de l'expansion rapide des plantations d'arbres est perçu comme positif ou neutre par les populations locales, malgré son impact négatif reconnu par la communauté scientifique sur la biodiversité au niveau du paysage (Fox et al., 2014; Chen et al., 2016). Plus généralement, nos résultats révèlent des perceptions divergentes entre acteurs locaux d'une part, et scientifiques et développeurs de l'autre, concernant l'impact des dynamiques agraires sur les services écosystémiques.

\subsection{Simulations participatives de mécanismes de PSE}

Les simulations de PSE ont intégré des «interventions REDD + virtuelles » de nature différente, selon qu'elles étaient réalisées en 2000 au début de la transition agraire, ou en 2012 une fois que la transition était bien avancée dans les différents pays. Ainsi, en présence de réserves forestières conséquentes, la marge de manœuvre des populations locales était plus importante en 2000, favorisant un engagement sur des pratiques de préservation de biomasses forestières à l'échelle de leur territoire villageois. Des parcelles forestières étaient mises en défens dans tous les villages d'étude et les revenus du PSE carbone étaient mobilisés pour financer l'intensification des terres agricoles (contrôle des animaux domestiques divagants et mise en place de pâturages améliorés, introduction de cultures commerciales, etc.), dont l'expansion était contrôlée dans le cadre d'un plan d'aménagement du territoire négocié collectivement. Par contre, en 2012, la marge de manœuvre était beaucoup plus réduite pour mettre en place des 
J-C Castella et G. Lestrelin : Cah. Agric. 2021, 30, 3

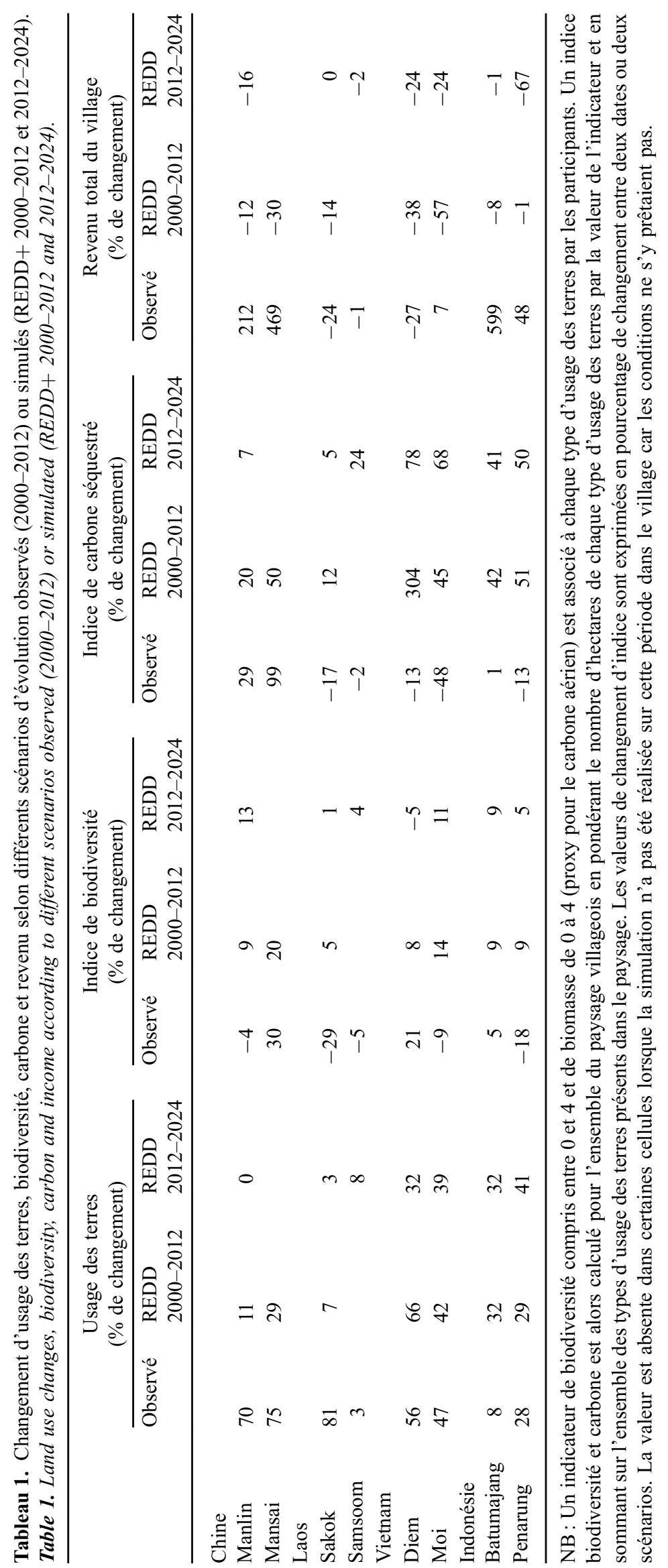

Page 7 de 11 
Tableau 2. Impact des changements d'usage des terres entre 2000 et 2012 sur les valeurs perçues des services écosystémiques dans les huit villages d'étude.

Table 2. Impact of land use changes between 2000 and 2012 on the perceived values of ecosystem services in the eight study villages.

\begin{tabular}{|c|c|c|c|c|c|c|c|c|c|c|c|c|}
\hline \multirow{3}{*}{\begin{tabular}{|l|} 
Pays \\
Chine
\end{tabular}} & \multirow{2}{*}{\begin{tabular}{|l|} 
Site \\
Manlin
\end{tabular}} & \multicolumn{2}{|c|}{ Moyens de subsistance } & \multicolumn{2}{|r|}{ Revenus } & \multicolumn{2}{|c|}{ Santé et médecine } & \multicolumn{2}{|c|}{ Tradition et culture } & \multicolumn{2}{|c|}{ Esthétique } & $\begin{array}{c}\text { Valeur totale des moyens } \\
\text { d'existence }\end{array}$ \\
\hline & & $\sqrt{2}$ & $-271 \%$ & 个 & $50 \%$ & 8 & $-19 \%$ & ת & $-174 \%$ & ת & $-20 \%$ & 1) $\quad 15 \%$ \\
\hline & Mansai & Љ & $-329 \%$ & $\hat{\imath}$ & $64 \%$ & § & $-47 \%$ & § & $-323 \%$ & 论 & $40 \%$ & $20 \%$ \\
\hline \multirow{2}{*}{ Laos } & Sakok & ᄀ & $-21 \%$ & $\Rightarrow$ & $-3 \%$ & ク & $-44 \%$ & $\hat{r}$ & $13 \%$ & $\hat{\imath}$ & $19 \%$ & $-9 \%$ \\
\hline & Samsoom & Љ & $-26 \%$ & ת & $-20 \%$ & $\Rightarrow$ & $-6 \%$ & $\Rightarrow$ & $-2 \%$ & $\Rightarrow$ & $8 \%$ & $-17 \%$ \\
\hline \multirow{2}{*}{ Vietnam } & Diem & $\sqrt{2}$ & $-96 \%$ & $\sqrt{2}$ & $-64 \%$ & ᄀ & $-42 \%$ & $\sqrt{2}$ & $-81 \%$ & $\sqrt{2}$ & $-69 \%$ & $-62 \%$ \\
\hline & Moi & $\Rightarrow$ & $-3 \%$ & $\Rightarrow$ & $-9 \%$ & ᄀ & $-14 \%$ & $\Rightarrow$ & $3 \%$ & $\sqrt{2}$ & $-37 \%$ & $-10 \%$ \\
\hline \multirow{2}{*}{ Indonésie } & Batumajang & ת & $-28 \%$ & ת & $-31 \%$ & \ & $-9 \%$ & $\sqrt{3}$ & $-8 \%$ & $\Rightarrow$ & $-3 \%$ & $-12 \%$ \\
\hline & Pearung & $\sqrt{2}$ & $-11 \%$ & $\eta$ & $-17 \%$ & ᄀ & $-15 \%$ & ᄀ & $-13 \%$ & $\sqrt{2}$ & $-15 \%$ & $-14 \%$ \\
\hline Pays & Site & \multicolumn{2}{|c|}{ Régulation hydrique } & \multicolumn{2}{|c|}{ Conservation des sols } & \multicolumn{2}{|c|}{ Biodiversité } & \multicolumn{2}{|c|}{ Qualité de l'air et climat } & \multicolumn{2}{|c|}{$\begin{array}{c}\text { Valeur totale des services } \\
\text { écosystémiques }\end{array}$} & $\begin{array}{l}\text { Valeur totale du paysage } \\
\text { (moyens d'existence et } \\
\text { services écosystémiques) }\end{array}$ \\
\hline \multirow{2}{*}{ Chine } & Manlin & ᄀ & $-39 \%$ & ת & $-52 \%$ & ת & $-42 \%$ & $\sqrt{3}$ & $-24 \%$ & 亿 & $-40 \%$ & $-5 \%$ \\
\hline & Mansai & $\Rightarrow$ & $2 \%$ & 个 & $33 \%$ & そ & $-189 \%$ & 个 & $35 \%$ & $\hat{\imath}$ & $10 \%$ & $18 \%$ \\
\hline \multirow{2}{*}{ Laos } & Sakok & $\hat{\imath}$ & $44 \%$ & 1ิ & $39 \%$ & 순 & $39 \%$ & $\hat{\imath}$ & $44 \%$ & 숭 & $42 \%$ & $24 \%$ \\
\hline & Samsoom & $\hat{\imath}$ & $17 \%$ & $\Rightarrow$ & $7 \%$ & 순 & $12 \%$ & 숭 & $26 \%$ & $\hat{\imath}$ & $14 \%$ & $-7 \%$ \\
\hline \multirow{2}{*}{ Vietnam } & Diem & Љ & $-40 \%$ & 8 & $-49 \%$ & Љ & $-44 \%$ & ᄀ & $-44 \%$ & § & $-43 \%$ & $-52 \%$ \\
\hline & Moi & 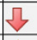 & $-35 \%$ & 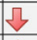 & $-21 \%$ & $\Rightarrow$ & $1 \%$ & ת & $-36 \%$ & \& & $-24 \%$ & $-17 \%$ \\
\hline \multirow{2}{*}{ Indonésie } & Batumajang & $\Rightarrow$ & $-1 \%$ & $\Rightarrow$ & $0 \%$ & $\Rightarrow$ & $-2 \%$ & $\Rightarrow$ & $-2 \%$ & $\Rightarrow$ & $-1 \%$ & $-6 \%$ \\
\hline & Pearung & Љ & $-20 \%$ & R & $-18 \%$ & Љ & $-19 \%$ & \ & $-15 \%$ & 及 & $-19 \%$ & $-16 \%$ \\
\hline
\end{tabular}

NB : Les calculs sont basés sur les valeurs perçues des moyens d'existence et des services écosystémiques associés à différents types d'usage des terres et au pourcentage de changement entre 2000 et 2012 obtenu grâce à la cartographie participative. La valeur totale sur l'ensemble du paysage est calculée en pondérant les surfaces des différents types d'usage des terres par les valeurs perçues issues du vote distributif (Sheil et Liswanti, 2006). Les flèches rouges indiquent une tendance à la baisse, vertes à la hausse et orange une stabilité sur la période considérée.

mécanismes REDD+. En particulier, le montant des crédits carbone était insuffisant pour être incitatif dans des contextes où la surface agricole occupait l'essentiel de l'espace villageois et où la forêt naturelle avait été remplacée par les plantations d'hévéa (Chine) ou des concessions forestières, mines ou plantations de palmier à huile (Indonésie). Au Laos et au Vietnam, les populations locales étant exclues de la gestion de larges surfaces forestières classées en zone de parc national, elles ne pouvaient plus bénéficier de crédits carbone du fait de la sortie des forêts naturelles de leur juridiction.

Dans tous les cas, un projet REDD+ initié en 2012 se serait traduit par une réduction des revenus villageois (Tab. 1), qu'il aurait été extrêmement difficile de compenser par la seule utilisation de crédits carbone, étant donné la biomasse forestière généralement limitée, les coûts d'opportunité élevés des cultures alternatives en Chine et en Indonésie, et l'absence d'additionnalité dans les cas du Laos et du Vietnam où des politiques de protection des forêts avaient déjà été appliquées. En bref, les fenêtres d'opportunité pour des mécanismes REDD+ se sont fermées très rapidement sous l'effet combiné de l'expansion des cultures de rente au détriment des forêts secondaires et de la mise en défens des forêts préservées, sous l'autorité des Etats (classées réserves naturelles) dans les années 2000. De plus, les nombreux problèmes de gouvernance liés à la technocratisation de la mise en œuvre (Angelsen et al., 2019; Ramcilovic-Suominen et Nathan, 2020; Vongvisouk et al., 2016) et les coûts de transaction élevés des programmes REDD+, qui s'ajoutent à des coûts d'opportunité déjà considérables (Lestrelin et al., 2019), ont mis à mal les perspectives de PSE REDD+ sur nos terrains d'études.

\section{Discussion}

\subsection{Implication des populations locales dans l'évaluation des services écosystémiques}

Les évaluations ex-ante des projets de PSE ont été possibles grâce à la traduction locale des différents services écosystémiques d'approvisionnement, de régulation, etc. et à leur évaluation participative engageant différents groupes d'acteurs. Notre étude a montré l'utilité des services écosystémiques pour évaluer des transformations paysagères à travers de multiples perspectives: sociales, économiques, culturelles, environnementales, etc. Danielsen et al. (2013) ont montré que les acteurs locaux sont à même de mesurer et de comparer les caractéristiques de différents environnements forestiers en termes de séquestration de carbone. Nous montrons ici qu'il en est de même pour d'autres services écosystémiques, dont l'évolution au cours du temps a pu être comparée selon différents scénarios. L'implication des acteurs dans des approches délibératives permet de faire émerger des consensus autour des préférences des populations locales en termes de trajectoires agraires et de définir les scénarios désirables de leur point de vue (Barnaud et Antona, 2014). Bien que les consensus obtenus lors de tels ateliers ne résistent généralement pas au passage à l'action collective, ils ont tout de même montré que la transition agraire avait privilégié les services d'approvisionnement au détriment des services de régulation et que les acteurs locaux étaient bien conscients des déséquilibres écosystémiques engendrés par ces transformations. 


\subsection{Les fenêtres d'opportunité REDD+ se sont refermées dans les années 2000}

Les résultats présentés dans le tableau 1 montrent que des initiatives REDD+ auraient eu une influence très limitée sur les changements d'usage des terres dans les années 2010, notamment dans le cas de la Chine où les paysages villageois étaient déjà largement convertis aux cultures de rente pérennes. $\mathrm{Au} \mathrm{Laos,} \mathrm{les} \mathrm{restrictions} \mathrm{d'accès} \mathrm{aux} \mathrm{espaces} \mathrm{forestiers,}$ imposées de longue date par le pouvoir politique, avaient déjà joué le rôle environnemental d'un projet REDD+ (sans compensation pour les populations locales privées d'accès aux ressources forestières). Dans le cas du Vietnam, la fenêtre d'opportunité d'un hypothétique programme REDD+ s'est refermée dans les années 2000, comme le montre l'impact réduit sur le paysage d'un projet REDD+ en 2012 par rapport à 2000 (Tab. 1). Les politiques de développement rural des années 1990 et 2000 se sont, là aussi, organisées autour de la délimitation d'aires forestières protégées et de l'attribution individuelle de terres agricoles. Elles ont ainsi réduit la pertinence d'un projet REDD+, qui dépendrait de crédits carbone très limités en raison de la faible biomasse des couvertures forestières restant sous gestion des communautés locales (Ankersen et al., 2015).

Dans les villages indonésiens, les territoires villageois sont dix fois plus vastes que dans les autres villages situés en Asie du Sud-Est continentale, et les densités de population y sont beaucoup plus faibles. Les menaces de déforestation de la part des populations locales sont donc très faibles et ne justifient pas économiquement un programme REDD+ centré sur les populations forestières. Enfin, si les grandes entreprises impliquées dans l'extraction du bois ou les plantations d'hévéas et de palmiers à huile constituent effectivement les principaux moteurs de la déforestation dans ces zones, les coûts d'opportunité sont extrêmement élevés par rapport à la valeur des émissions carbone qui peuvent être évitées par un programme REDD+.

\subsection{Une évolution nécessaire des mécanismes de PSE}

Malgré les contraintes mentionnées ci-dessus, des initiatives PSE de type REDD+ ont déjà été mises en œuvre en Chine (He, 2014) et au Vietnam (Clement et Amezaga, 2009). Ces initiatives reposent clairement sur une combinaison de politiques nationales et d'adaptations locales. La priorité n'est plus à la compensation de coûts d'opportunités, irréaliste étant donné la faible valeur des crédits carbone sur le marché mondial, ni à la rétribution d'un service mesuré en volume de carbone séquestré ou de déforestation évitée, irréaliste du fait des contraintes organisationnelles, techniques et logistiques pour évaluer l'écart à des scénarios de référence dans des contextes de gouvernance faible (Müller et al., 2014; Vongvisouk et al., 2016; Mertz et al., 2018). La priorité est encore moins à la prise en compte des co-bénéfices attendus en termes de capacité d'adaptation au changement climatique, par exemple biodiversité, sécurité alimentaire et réduction de la pauvreté, qui rendrait l'équation REDD+ encore plus complexe (Sills et al., 2014 ; Pasgaard et al., 2016).

Les approches juridictionnelles qui ont émergé ces dernières années (Boyd et al., 2018; Irawan et al., 2019) offrent une alternative pragmatique en appuyant les «bonnes pratiques», celles dont on peut attendre un impact positif sur les services écosystémiques sans engagement contraignant sur des performances ou des résultats difficilement mesurables. Les approches juridictionnelles reviennent donc à financer la REDD+ sur la base des moyens engagés pour fournir des services écosystémiques et non plus sur les impacts en termes de séquestration effective de carbone ou d'émission évitée par rapport à un scénario de référence. Dans ce contexte-là, les mécanismes de simulation participative sont utiles pour engager les populations locales et les autres acteurs de la REDD+ dans la co-conception de mécanismes locaux de mise en œuvre. L'approche présentée dans cet article pourrait aussi être utilisée pour évaluer l'impact potentiel de différentes pratiques selon les contextes locaux et les aspirations des différents groupes d'acteurs. Des simulations ex-ante des dynamiques de transformation des paysages permettraient d'identifier les leviers techniques et/ou organisationnels les mieux adaptés aux conditions locales.

\section{Conclusion}

Dans le contexte politique qui prévaut dans les pays postsocialistes tels que la Chine, le Laos et le Vietnam, il y a peu de chances qu'un processus ascendant ou des systèmes décentralisés de gestion des PSE soient mis en place de façon spontanée. Cependant, les populations locales déterminent dans une large mesure le succès de ces politiques et des mécanismes d'intervention qui leur sont associés. La méthode d'évaluation des services écosystémiques présentée dans cet article engage les communautés locales dans un processus collectif d'exploration de la pertinence des PSE, de leur impact local et de modes d'intervention alternatifs. Elle a montré, au travers d'une série d'expérimentations participatives, que les mécanismes REDD+ tels qu'ils avaient été conçus initialement, i.e. compensation de coûts d'opportunité associée à des changements d'usage des terres évitant la déforestation ou la dégradation forestière, arrivent trop tard pour inverser les changements agraires des années 2000 (Müller et al., 2014 ; Lestrelin et al., 2019). Les coûts de transaction sont tellement élevés et la gouvernance des dispositifs PSE si complexe, que la forêt a presque entièrement disparu sous l'influence de l'expansion agricole avant que le dispositif n'ait pu être mis en place. Après plus de dix ans de projets préparatoires et d'études de faisabilité en tous genres (Minang et al., 2014), l'une des principales leçons tirées de cette étude est que l'évaluation de la faisabilité des mécanismes REDD+ va bien au-delà de l'étude de la compensation potentielle des coûts d'opportunité (Lestrelin et al., 2019). Si l'on considère uniquement les coûts d'opportunité, REDD + ne serait réalisable que dans des zones très limitées d'Asie du Sud-Est et aurait donc très peu d'impact sur le changement climatique et, bien entendu, sur les autres services écosystémiques. Ajouter trop d'objectifs amène à perdre de vue l'intention initiale de réduction des émissions de $\mathrm{CO}_{2}$ et à rendre la mise en œuvre des mécanismes REDD+ difficile sur le terrain (Angelsen et al., 2019). Des approches juridictionnelles ont été développées pour promouvoir la transition vers des paysages multifonctionnels, mais elles sont confrontées aux mêmes contraintes technocratiques de mise en œuvre que les projets REDD+ de première génération. Finalement, en perdant sa spécificité liée au service 
écosystémique carbone, le programme REDD+ s'est éloigné de ses ambitions initiales pour devenir un instrument supplémentaire de développement durable.

Remerciements. Les auteurs tiennent à remercier Thoumthone Vongvisouk (Faculty of Forest Sciences, National University of Laos, Vientiane), Nguyen Dinh Tien (Faculty of Development Economics, Vietnam National University, Hanoi), Janudianto (World Agroforestry Centre ICRAF, Bogor), Qiaohong Li (Kunming Institute of Botany, Chinese Academy of Sciences, Kunming) pour leur accompagnement et leur appui décisif durant les activités de terrain. Nous tenons aussi à exprimer notre profonde gratitude à Ole Mertz (Department of Geosciences and Natural Resource Management, University of Copenhagen, Copenhague), qui a assuré la coordination du projet de recherche intitulé «Impacts of Reducing Emissions from Deforestation and Forest Degradation and Enhancing Carbon Stocks (I-REDD+)», d'où sont issus les résultats de terrain présentés dans cet article. I-REDD+ était financé par la Commission européenne (Grant no. 265286).

\section{Références}

Angelsen A, Martius C, De Sy V, Duchelle AE, Larson AM, Pham TT (ed). 2019. REDD+ : la transformation. Enseignements et nouvelles directions. Bogor, Indonésie : CIFOR, 303 p. DOI: 10.17528/cifor/007447.

Ankersen J, Mertz O, Fensholt R, Castella JC, Lestrelin G, Nguyen TD et al. 2015. Vietnam's forest transition in retrospect: demonstrating weaknesses in business-as-usual scenarios for REDD+. Environmental Management 55(5): 1080-1092. DOI: 10.1007/s00267-015-0443-y.

Barbier EB, Tesfaw AT. 2012. Can REDD+ Save the Forest? The Role of Payments and Tenure. Forests 3(4): 881-995. DOI: 10.3390/f3040881.

Barnaud C, Antona M. 2014. Deconstructing ecosystem services: uncertainties and controversies around a socially constructed concept. Geoforum 56: 113-123. DOI: 10.1016/j.geofo rum.2014.07.003.

Bourgoin J, Castella JC, Pullar D, Lestrelin G, Bouahom B. 2012. Toward a land zoning negotiation support platform: "Tips and tricks" for participatory land use planning in Laos. Landscape and Urban Planning 104: 270-278. DOI: 10.1016/j.landurb plan.2011.11.008.

Bourgoin J, Castella JC, Hett C, Lestrelin G, Heinimann A. 2013. Engaging local communities in low emissions land use planning: a case study in Laos. Ecology and Society 18(2): 9. DOI: 10.5751/ ES-05362-180209.

Boyd W, Stickler C, Duchelle AE, Seymour F, Nepstad D, Bahar NHA et al. 2018. Jurisdictional Approaches to REDD+ and Low Emissions Development: Progress and Prospects. Working Paper. Washington, DC: World Resources Institute, 14 p. wri.org/endingtropicaldeforestation.

Brofeldt S, Theilade I, Burgess ND, Danielsen F, Poulsen MK, Adrian $\mathrm{T}$ et al. 2014. Community Monitoring of Carbon Stocks for REDD+: Does Accuracy and Cost Change over Time? Forests 5 (8): 1834-1854. DOI: 10.3390/f5081834.

Bruun TB, Berry N, de Neergaard A, Xaphokahme P, McNicol I, Ryan CM. 2018. Long rotation swidden systems maintain higher carbon stocks than rubber plantations. Agriculture, Ecosystems and Environment 256: 239-249. DOI: 10.1016/j.agee.2017.09.010.
Castella JC, Lestrelin G, Hett C, Bourgoin J, Fitriana YR, Heinimann A et al. 2013. Effects of landscape segregation on livelihood vulnerability: Moving from extensive shifting cultivation to rotational agriculture and natural forests in northern Laos. Human Ecology 41(1): 63-76. DOI: 10.1007/s10745-012-9538-8.

Castella JC, Bourgoin J, Lestrelin G, Bouahom B. 2014. A model of the science-practice-policy interface in participatory land-use planning: lessons from Laos. Landscape Ecology 29(6): 10951107. DOI: 10.1007/s10980-014-0043-x.

Cerbu G, Minang P, Swallow B, Meadu V. 2009. Global survey of REDD projects: What implications for global climate objectives? ASB Policy Brief 12. Nairobi: ASB Partnership for the Tropical Forest Margins, 4 p. www.asb.cgiar.org/PDFwebdocs/ASBPB12. pdf.

Chen H, Yi ZF, Schmidt-Vogt D, Ahrends A, Beckschäfer P, Kleinn C et al. 2016. Pushing the limits: The pattern and dynamics of rubber monoculture expansion in Xishuangbanna, SW China. PLoS ONE 11: e0150062. DOI: 10.1371/journal.pone.0150062.

Chhatre A, Agrawal A. 2009. Trade-offs and synergies between carbon storage and livelihood benefits from forest commons. Proceedings of the National Academy of Sciences of the United States of America 106(42): 17667-17670. DOI: 10.1073/ pnas.0905308106.

Clement F, Amezaga JM. 2009. Afforestation and forestry land allocation in northern Vietnam: Analysing the gap between policy intentions and outcomes. Land Use Policy 26(2): 458-470. DOI: 10.1016/j.landusepol.2008.06.003.

Cramb RA, Colfer CJP, Dressler W, Laungaramsri P, Trung LQ, Mulyoutami E et al. 2009. Swidden Transformations and Rural Livelihoods in Southeast Asia. Human Ecology 37: 323-346. DOI: 10.1007/s10745-009-9241-6.

Danielsen F, Adrian T, Brofeldt S, van Noordwijk M, Poulsen MK, Rahayu S et al. 2013. Community monitoring for REDD+: international promises and field realities. Ecology and Society 18 (3): 41. DOI: 10.5751/ES-05464-180341.

Fox J, Castella JC, Ziegler AD. 2014. Swidden, rubber and carbon: Can REDD+ work for people and the environment in Montane Mainland Southeast Asia? Global Environmental Change 29: 318326. DOI: 10.1016/j.gloenvcha.2013.05.011.

Grainger A, Boucher DH, Frumhoff PC, Laurance WF, Lovejoy T, McNeely J et al. 2009. Biodiversity and REDD at Copenhagen. Current Biology 19: 974-976. DOI: 10.1016/j.cub.2009.10.001.

He J. 2014. Governing forest restoration: Local case studies of sloping land conversion program in Southwest China. Forest Policy and Economics 46: 30-38. DOI: 10.1016/j.forpol.2014.05.004.

Irawan S, Widiastomo T, Tacconi L, Watts JD, Steni B. 2019. Exploring the design of jurisdictional REDD+: The case of Central Kalimantan, Indonesia. Forest Policy and Economics 108: 101853. DOI: 10.1016/j.forpol.2018.12.009.

Jindal R, Swallow B, Kerr J. 2008. Forestry-based carbon sequestration projects in Africa: Potential benefits and challenges. Natural Resources Forum 32: 116-130. DOI: 10.1111/ j.1477-8947.2008.00176.x.

Leggett M, Lovell H. 2012. Community perceptions of REDD+: a case study from Papua New Guinea. Climate Policy 12(1): 115124. DOI: 10.1080/14693062.2011.579317.

Lestrelin G, Trockenbrodt M, Phanvilay K, Thongmanivong S, Vongvisouk T, Pham TT et al. 2013. The context of REDD+ in the Lao People's Democratic Republic: Drivers, agents and institutions. Occasional Paper 92. Bogor, Indonesia: CIFOR.

Lestrelin G, Castella JC, Li Q, Vongvisouk T, Nguyen DT, Mertz O. 2019. A nested land uses-landscapes-livelihoods approach to 
assess the real costs of land-use transitions: Insights from southeast Asia. Land 8(1): 11. DOI: 10.3390/land8010011.

Matthews R, Van Noordwijk M. 2014. From euphoria to reality on efforts to reduce emissions from deforestation and forest degradation (REDD+). Mitigation and Adaptation Strategies for Global Change 19: 615-620. DOI: 10.1007/s11027-014-9577-0.

Mertz O. 2009. Trends in shifting cultivation and the REDD mechanism. Current Opinion in Environmental Sustainability 1: 156-160. DOI: 10.1016/j.cosust.2009.10.002.

Mertz O, Padoch C, Fox J, Cramb RA, Leisz SJ, Nguyen TL et al. 2009. Swidden change in Southeast Asia: understanding causes and consequences. Human Ecology 37: 259-264. DOI: 10.1007/ s10745-009-9245-2.

Mertz O, Grogan K, Pflugmacher D, Lestrelin G, Castella JC, Vongvisouk $\mathrm{T}$ et al. 2018. Uncertainty in establishing forest reference levels and predicting future forest-based carbon stocks for REDD+. Journal of Land Use Science 13: 1-15. DOI: 10.1080/ 1747423X.2017.1410242.

Minang PA, Van Noordwijk M, Duguma LA, Alemagi D, Do TH, Bernard F et al. 2014. REDD+ Readiness progress across countries: time for reconsideration. Climate Policy 14(6): 685708. DOI: 10.1080/14693062.2014.905822.

Müller D, Sun Z, Vongvisouk T, Pflugmacher D, Xu J, Mertz O. 2014. Regime shifts limit the predictability of land-system change. Global Environmental Change 28: 75-83. DOI: 10.1016/j. gloenvcha.2014.06.003.

Pasgaard M, Sun Z, Müller D, Mertz O. 2016. Challenges and opportunities for REDD+: A reality check from perspectives of effectiveness, efficiency and equity. Environmental Science \& Policy 63: 161-169. DOI: 10.1016/j.envsci.2016.05.021.

Pham TT, Castella JC, Lestrelin G, Mertz O, Le D, Moeliono M et al. 2015. Adapting Free, Prior, and Informed Consent (FPIC) to Local
Contexts in REDD+: Lessons from Three Experiments in Vietnam. Forests 6: 2405-2423. DOI: 10.3390/f6072405.

Pham TT, Hoang TL, Nguyen DT, Dao TLC, Ngo HC, Pham VH. 2019. The context of REDD+ in Vietnam: Drivers, agents and institutions (2nd edition). Occasional Paper 196. Bogor, Indonesia: CIFOR.

Ramcilovic-Suominen S, Nathan I. 2020. REDD+ policy translation and storylines in Laos. Journal of Political Ecology 27(1): 1-20. DOI: $10.2458 / \mathrm{V} 27 \mathrm{I} 1.23188$.

Schmidt-Vogt D, Leisz S, Mertz O, Heinimann A, Thiha T, Messerli P et al. 2009. An assessment of trends in the extent of swidden in Southeast Asia. Human Ecology 37: 269-280. DOI: 10.1007/ s10745-009-9239-0.

Sheil D, Liswanti N. 2006. Scoring the importance of tropical forest landscapes with local people: patterns and insights. Environmental Management 38: 126-136. DOI: 10.1007/s00267-005-0092-7.

Sills EO, Atmadja SS, de Sassi C, Duchelle AE, Kweka DL, Resosudarmo IAP et al. 2014. REDD+ on the ground. A casebook of subnational initiatives across the globe. Bogor, Indonesia: CIFOR, $505 \mathrm{p}$.

Vongvisouk T, Lestrelin G, Castella JC, Mertz M, Brandt Broegaard R, Thongmanivong S. 2016. REDD+ on hold: Lessons from an emerging institutional setup in Laos. Asia Pacific Viewpoint 57(3): 393-405. DOI: 10.1111/apv.12134.

Wunder S, Wertz-Kanounnikoff S. 2009. Payments for Ecosystem Services: A New Way of Conserving Biodiversity in Forests. Journal of Sustainable Forestry 28: 576-596. DOI: 10.1080/ 10549810902905669.

Wunder S, Engel S, Pagiola S. 2008. Taking stock: A comparative analysis of payments for environmental services programs in developed and developing countries. Ecological Economics 65: 834-852. DOI: 10.1016/j.ecolecon.2008.03.010.

Citation de l'article : Castella J-C, Lestrelin G. 2021. Explorer l'impact environnemental des transformations agraires en Asie du Sud-Est grâce à l'évaluation participative des services écosystémiques. Cah. Agric. 30: 3. 